\title{
The accuracy of pre-operative digital templating in total hip arthroplasty performed in a low-volume, resource-constrained orthopaedic unit
}

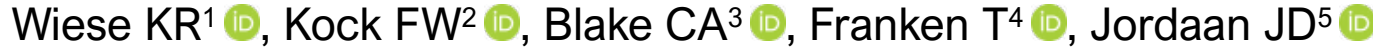 \\ 1 MBChB; Registrar, Division of Orthopaedic Surgery, Stellenbosch University, South Africa \\ 2 MBChB; Registrar, Division of Orthopaedic Surgery, Stellenbosch University, South Africa \\ 3 MBChB, MMed(Orth); Consultant, Department of Orthopaedic Surgery, University of the Free State, Bloemfontein, South Africa \\ 4 MBChB, MMed(Orth); Consultant, Department of Orthopaedic Surgery, Worcester Provincial Hospital; Division of Orthopaedic Surgery, Stellenbosch \\ University, South Africa \\ 5 MBChB, H Dip Orth(SA), FC Orth(SA); Orthopaedic surgeon, Division of Orthopaedic Surgery, Stellenbosch University, South Africa
}

Corresponding author: Dr Karin Rae Wiese, Division of Orthopaedic Surgery, Department of Surgical Sciences,

Faculty of Medicine and Health Sciences, Stellenbosch University, South Africa; tel: 021938 4911; email: karinraewiese@gmail.com

\begin{abstract}
Aims: Total hip arthroplasty (THA) is considered one of the most successful surgical procedures in modern medicine. The success of THA is well documented, and includes high patient satisfaction rates, low morbidity rates and cost-effective surgery. Most publications come from THA performed in high-volume arthroplasty units, done in high-income countries. Limited data is available on THA performed in low-volume, low-income countries. The aim of this study was to evaluate the accuracy of digital templating in a low-volume, resource-constrained orthopaedic unit from 2016 to 2017. We introduced a standardised hip radiography programme, followed by a stepwise pre-operative templating method. We compared the implant sizes inserted during THA with the templated sizes determined pre-operatively. This was to deduct whether digital templating in a low-volume arthroplasty unit is accurate and of the same value as digital templating done in a high-volume unit.

Methods: A descriptive retrospective study was conducted on all patients who received elective primary uncemented THA in a low-volume, resource-constrained orthopaedic unit. Pre-operative radiographs were done according to guidelines published by Scheerlinck followed by pre-operative templating using the Impax Orthopaedic tools $®$ software and a stepwise technique described by Bono. Implanted prosthesis sizes, as recorded in operation notes, were retrospectively compared to pre-operative templating.

Results: A total of 56 participants were included (30 females, 26 males), with a mean age of 55.5 (32-78) years. On the acetabular side, in $71 \%(n=40 ; p<0.001)$ there was a cumulative difference of one implant size between the templated cup size and the actual cup size used. On the femoral side, in $79 \%(n=44 ; p<0.001)$ there was a cumulative difference of one implant size between the templated stem size and the actual stem size used. Oversizing of the implants was more prevalent, with $20 \%(n=11)$ of the acetabular components oversized by two or more sizes and $13 \%(n=7)$ of the femoral components oversized by two or more sizes.

Conclusion: With the introduction of a standardised radiology programme and a stepwise templating technique, the benefits and accuracy of pre-operative templating done in a low-volume, resource-constrained orthopaedic unit is comparable to published data done in high-volume arthroplasty units.
\end{abstract}

Level of evidence: Level 4

Keywords: arthroplasty, templating, calibration, radiographs, implant, acetabulum

Citation: Wiese KR, Kock FW, Blake CA, Franken T, Jordaan JD. The accuracy of pre-operative digital templating in total hip arthroplasty performed in a low-volume, resource-constrained orthopaedic unit. SA Orthop J 2020;19(1):28-32. http://dx.doi.org/10.17159/2309-8309/2020/v19n1a4

Editor: Dr M Held, University of Cape Town, Cape Town, South Africa

Received: September 2018

Accepted: August 2019

Published: March 2020

Copyright: $\odot 2020$ Wiese KR, et al. This is an open-access article distributed under the terms of the Creative Commons Attribution Licence, which permits unrestricted use, distribution and reproduction in any medium, provided the original author and source are credited.

Funding: No external funding received.

Conflict of interest: All authors declare there are no conflicts of interest with regard to this study. 


\section{Introduction}

Total hip arthroplasty (THA) is considered one of the most successful surgical procedures in modern medicine. ${ }^{1}$ Treatment goals include pain relief, restoration of normal hip biomechanics and improvement in function. ${ }^{1}$ The success of THA is well documented, with high patient satisfaction rates, low morbidity rates and cost-effective surgery. ${ }^{2}$ Most of these publications come from THA performed in high-volume arthroplasty units, done in high-income countries. Limited data is available on THA performed in low-volume, low-income countries.

Pre-operative templating for THA is considered an essential part of planning prior to performing hip arthroplasty. Pre-operative templating is highly beneficial to both the surgeon and ultimately the patient as it alerts the surgeon to the need for unusual sized components or additional instruments and therefore allows for a smooth and well-planned surgery.,4 Pre-operative templating also reduces the intra-operative stress experienced by the surgeon and aids the surgeon to ensure correction of hip biomechanics. Failure to obtain adequate femoroacetabular offset or the correction of limb length discrepancy following THA results in altered hip biomechanics, and thus influences the patient's functional outcome and the longevity of the implant. ${ }^{3,4}$ Both Charnley and Müller advocated for the use of pre-operative templating as it encourages the surgeon to think three-dimensionally, improve the precision of the surgery, reduce the length of the procedure and reduce the incidence of post-operative complications. ${ }^{5}$

Digital radiography has replaced traditional radiography in most healthcare facilities worldwide. ${ }^{6}$ This led to the development and use of computer-based templating programmes instead of standard onlay templating techniques. These templating programmes rely on a calibration device in order to template accurately. For example, the use of a $20 \mathrm{~mm}$ radiographic marker such as a metal ball bearing, placed in a clear plastic tube, positioned at the same depth as the greater trochanter has proven to be a cost-effective, accurate and repeatable method of calibrating the size of digital radiographs..$^{7,8}$ The contralateral hip is often used for templating as it is often less affected by a pathological process and offers a mirror image of the planned surgical field. ${ }^{7}$ It is thus essential to be able to see both hips on the AP pelvis radiograph.

Due to high costs, digital templating software is not readily available in units that perform THA in low-income countries. Currently, we were unable to find any studies that analyse the accuracy of pre-operative templating in a low-volume, low-income country where THA is performed in a resource-constrained orthopaedic unit.

The exact number to define the term 'high- and/or low-volume arthroplasty unit' is not well defined in the literature. The most accepted number for a high-volume arthroplasty unit, as described by Katz et al. of $>100$ procedures per year was used in our study. ${ }^{9}$

The surgical technique and intra-operative considerations are different for cemented and uncemented THA. In uncemented THA, the accuracy of the exact implant size is very important, either to prevent peri-operative fractures from oversized implants or subsidence from undersized implants. In cemented THA, the cement and cement mantle allow for a more forgiving implant size selection..$^{3,4}$ For this reason, we specifically studied the data for uncemented implants.

The aim of this study was to evaluate the accuracy of digital templating in a low-volume, resource-constrained orthopaedic unit from 2016 to 2017 . We introduced a standardised hip radiography programme, followed by a stepwise pre-operative templating method. We compared the actual implant sizes, cup and stem, inserted during THA with the templated implant sizes determined pre-operatively. This was to deduce whether digital templating in a low-volume arthroplasty unit is accurate and of the same value as digital templating done in a high-volume unit.

\section{Materials and methods}

A descriptive retrospective review was performed from 1 January 2016 to 31 December 2017 on all patients who underwent elective uncemented THA at a low-volume, secondary-level provincial hospital in South Africa that has significant resource constraints. Exclusion criteria included traumatic neck of femur fractures requiring THA, cemented total hip arthroplasty and hybrid systems.

A total of 109 THA surgeries were performed during the two-year review period, thus on average $<100$ surgeries per year. Of the 109 THA performed, 53 cases were excluded for neck of femur fracture cases, including cemented or hybrid THA procedures.

A total of 56 elective primary uncemented total hip arthroplasties were performed during this time period. Medical records, including admission notes, operation reports and radiographs were retrospectively reviewed.

Pre-operative radiographs were done according to guidelines published by Scheerlinck. ${ }^{10}$ This included standard anteroposterior (AP) view radiographs of the pelvis and proximal femurs as well as a lateral view of the affected hip. On the radiographs, visualisation of the proximal one-third of the femur on the AP pelvis view facilitated templating of the femoral component.

Pre-operative templating was done by the primary surgeon, or the assisting medical officer, using the Impax Orthopaedic Tools ${ }^{\circledR}$ software. During the pre-operative planning ward round, the templating was reviewed and the final decision was saved in the patient records.

All THAs were performed by a senior orthopaedic surgeon through a standard anterolateral approach. DuPuy Synthes ${ }^{\circledR}$ uncemented implants, the Pinnacle cup and Summit stem (Le Locle, Switzerland) were used for all cases.

During surgery the surgeon had access to both the electronic recording of the pre-operative templating as well as the documented implant sizes. The final implant sizes were chosen based on clinical operative feedback by the surgeon. The final implant sizes were then recorded in the operative notes. This data was analysed retrospectively and compared to the pre-operative templated size.

\section{Standardised radiograph technique}

Pre-operative radiographs were standardised according to the technique described by Scheerlinck. ${ }^{10}$ This included a standing AP pelvis and lateral hip radiograph.

The AP pelvis radiograph is taken in the standing position with both feet internally rotated 15 degrees. Both iliac spines are placed at the same distance from the film to standardise the radiographs and ensure adequate visualisation of the femoral neck length. The radiograph beam is centred on the symphysis pubis rather than the sacrum and this is to ensure inclusion of the proximal third of the femur, which is required to perform templating of the femoral stem component.

All of the pre-operative radiographs were obtained with a standardised $100 \mathrm{~cm}$ distance from the radiograph tube to the radiograph plate. This resulted in an average magnification of $20 \% \pm 6 \% .6,10$ In the event that the radiograph was deemed to be of poor quality, the patient was sent for repeat radiographs.

Although we utilise both AP and lateral radiographs as part of our radiographic workup, for the purpose of this study, we only templated from the AP radiograph.

\section{Pre-operative templating}

The stepwise approach to digital pre-operative templating was utilised as described by Bono. ${ }^{11}$

Step 1 includes determining the magnification of the radiograph. This is achieved with the use of a $20 \mathrm{~mm}$ ball bearing marker 
inside a plastic tube (Figure 1). The marker is placed between the patient's legs, at the same depth as the greater trochanters for all pre-operative radiographs. The calibration software, Impax Orthopaedic Tools Planning Module Hip, uses this marker to calibrate the radiograph for templating (Figure 2).

Step 2 consists of determining the pelvic axis. This is achieved with the AP pelvis radiograph and the templating software, which utilises the acetabular teardrop as a landmark. A line connecting the left and right acetabular teardrops serves as a reference for pelvic orientation and from this reference point, left and right limb length corrections are then accomplished. The pelvic axis also determines the appropriate abduction or inclination angle of the acetabular component ensuring that the prosthesis is placed in the correct position relative to the pelvis.

Step 3 involves determining whether a limb length discrepancy is present. This is accomplished by a measurement from the tip of the lesser trochanter perpendicular to the pelvic axis as determined in step 2.

Step 4 is to determine the centre of rotation of the hip joint. The software achieves this by importing a digital acetabular template within the osseous confines of the acetabulum. It is important to ensure that the cup is imported centrally at a 45 degree angle to the pelvic axis.

Step 5 is to determine the size of the femoral component. This is done by measuring the width of the medullary canal within the proximal femoral diaphysis as well as at the metaphyseal flare. With the aid of the calibration software an appropriately sized femoral stem is then selected.

During step 6 the appropriately sized digital femoral component is placed within the femur in such a position as to reproduce the existing limb length or to correct for any limb length discrepancy. The combination of acetabular and femoral implants should reproduce the appropriate femoral offset of the hip with the ability to fine-tune this utilising different head length sizes.

Templating was routinely done during the pre-operative arthroplasty ward round by the planning surgeon. This was supervised and confirmed by the orthopaedic consultant after which it was saved on Impax Orthopaedic Tools for later use during surgery.

\section{Intra-operative decision-making}

Intra-operatively, the surgeon had access to the pre-operative templating. The final decision on sizing was however made on clinical grounds using standard operating techniques. At the time of the surgery, the surgeons were not aware of this retrospective study that followed later.

\section{Statistical analysis}

Basic descriptive statistics were reported including differences in implants (cup and stems) between pre-operative templating and intra-operative decision-making. Information such as age and sex was also recorded for basic demographic data. A chi-square test was used to detect differences between the number of participants where less than or equal to one change in template size was required compared to those who required a change of more than one size. Data was analysed using Excel and Statistica v13.

\section{Results}

The mean age of all participants $(n=56)$ was $55.5(32-78)$ years. Of these, $54 \%(n=36)$ of the participants were female and $46 \%(n=20)$ were male.

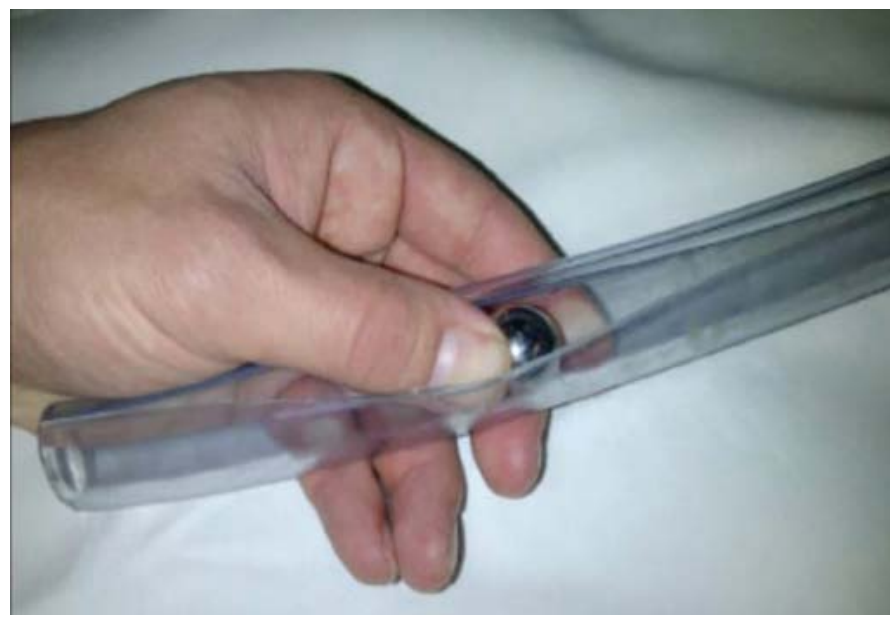

Figure 1. Ball bearing inside a clear plastic tube used as a marker for calibration of the radiograph

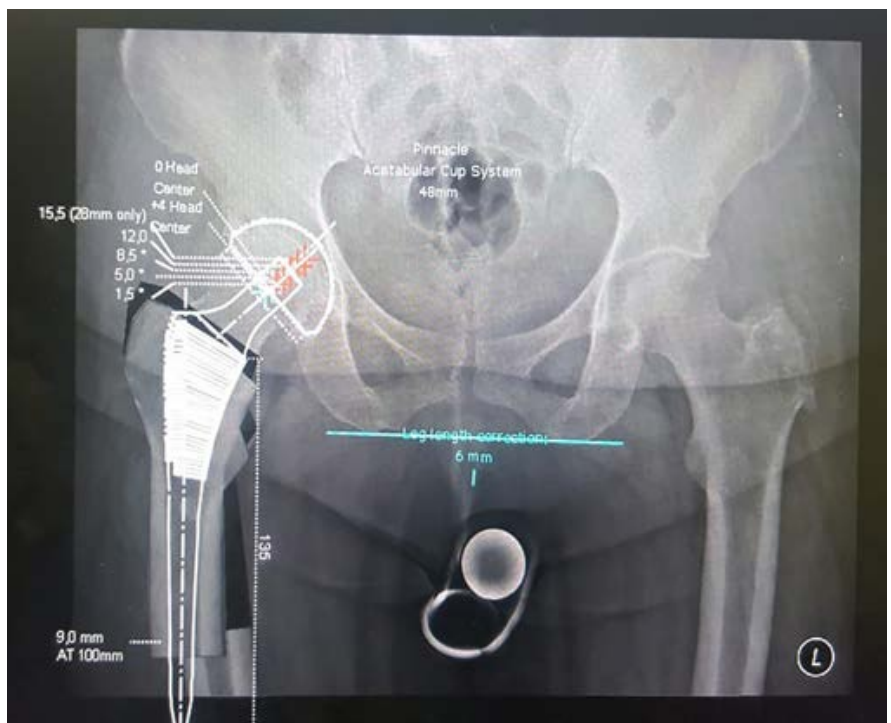

Figure 2. AP pelvis radiograph showing Impax Orthopaedic Tools Planning Module Hip calibration and templating software

\section{Acetabular components}

The median difference between implant size templated and actual implant size used was 1 (interquartile range $0-2$, range $0-3$ ). The exact acetabular cup size was predicted in $30 \%$ of cases $(n=17)$ (Table I). Cumulatively, in $71 \%$ of cases $(n=40)$, the implant inserted was within one implant size (above, equal or below) of the templated acetabular cup size. In $29 \%$ of cases ( $n=16)$, the implanted cup was more than one size above the templated size $(p<0.001)$.

Cumulatively in $98 \%(n=55)$ of the hips, acetabular components were within two sizes $(4 \mathrm{~mm})$ smaller, equal or larger than the templated size (Table I).

\section{Femoral stem component differences}

The median difference between implant size templated and actual implant size used was 1 (interquartile range $0-1$, range $-4-3$ ). The exact femoral stem size was also predicted in $30 \%(n=17)$ of cases (Table I). Cumulatively, in $79 \%$ of cases $(n=44)$, the implant inserted was within one implant size (above, equal or below) of the templated stem size. In $21 \%(n=12)$ the implanted stem was more than one size above or below the templated size $(p<0.001)$.

Cumulatively, in $96 \%(n=54)$ of the hips, the femoral stem components were within two implant sizes of the templated size (smaller, equal to or larger) (Table I). 


\section{Outliers}

In our study we found that we tend to oversize both the acetabular and femoral components during pre-operative digital templating. Twenty per cent $(n=11)$ of the acetabular components were oversized by two or more sizes, and $13 \%(n=7)$ of the femoral components were oversized by two or more sizes. Nine per cent $(n=5)$ of the acetabular and femoral components were undersized by two or more sizes, respectively.

Males were templated more accurately than females, with $17 \%$ $(n=9)$ of males compared to $32 \%(n=19)$ of females having an acetabular cup and femoral stem size differentiation of two or more between templated and implanted implant sizes.

\section{Discussion}

During the last decade, the indications for THA have broadened with an increase in patients' expectations in functional outcome and implant longevity. Improvements in technology have addressed both fixation and wear issues. ${ }^{10}$ The development of pre-operative templating has allowed surgeons to focus more attention on the optimisation of hip biomechanics and to anticipate the correct implant sizing, positioning, as well as potential difficulties prior to surgery. ${ }^{10}$

The majority of studies on THA, including pre-operative templating, are conducted in high-volume arthroplasty units. Katz et al. defined a high-volume arthroplasty unit as one in which $>100$ procedures are performed annually. ${ }^{9}$ These studies showed a significant reduction in complications following THA in higher volume units. This was due to pre-operative planning and surgeons being more comfortable and familiar with the procedure..$^{12}$

We could find no publication on pre-operative templating and its benefits in a low-volume arthroplasty unit. The opinion of the senior authors was that the benefits of pre-operative templating would be equal, if not better, to the surgeons who do not perform this procedure as regularly as high-volume surgeons.

The use of a stepwise approach to digital templating, such as that described by Bono, ensures a repeatable and reliable method. ${ }^{11}$ Pre-operative templating alerts the surgeon in difficult cases, where restoration of normal hip anatomy is not possible with standard implants, to request extended selections of implants to be available

Table I: Overview of templating size differences compared to in-situ implant sizes used

\begin{tabular}{|c|c|c|c|}
\hline Component & Size difference & Count (n) & Per cent \\
\hline Femoral & & & \\
\hline & -4 & 1 & 1.8 \\
\hline & -3 & 1 & 3.6 \\
\hline & -1 & 2 & 19.6 \\
\hline Acetabular & 0 & 11 & 30.4 \\
\hline & 1 & 17 & 28.6 \\
\hline & 2 & 8 & 14.3 \\
\hline & -3 & 1 & 1.8 \\
\hline & -2 & 10 & 17.9 \\
\hline & -1 & 14 & 25 \\
\hline
\end{tabular}

in theatre. This is important in low-volume units that do not carry a large in-hospital consignment of the full range of implants and/or revision implants. ${ }^{10}$

Anyaehie et al.'s study which focused on total knee arthroplasty in a resource-constrained environment, similar to that of the public health sector of South Africa, showed that the absence of ideal infrastructure and equipment alongside deficient surgical expertise are some of the reasons why many hospitals in resourceconstrained areas do not offer arthroplasty services. ${ }^{13}$ Patients in these environments were also shown to present much later for joint arthroplasty. Reasons for this included a preference for traditional healers; patients have easier access to their services compared to utilising hospital services with long waiting lists. Further contributing to delayed presentation are cultural beliefs, together with the health-seeking behaviour of patients who accept pain and disability until they become immobile before seeking arthroplasty services. These factors lead to complex pathology with severe joint degeneration on presentation, resulting in conservative treatment no longer being an option, with arthroplasty the only solution to improve pain and function. ${ }^{13}$

The recent increased use of collared implants may be a solution to this problem, with collared uncemented stems showing improved axial and rotational stability. This prevents subsidence and aids the stem to remain in its intra-operative position. ${ }^{10}$

Several studies have been published that aim to evaluate the accuracy and reliability of pre-operative templating, with results being favourable towards templating as shown by Knight and Atwater. Implants inserted during uncemented THA within one size above or below what was templated were deemed to be adequate. ${ }^{14}$

The use of pre-operative templating encourages the surgeon to think three-dimensionally and greatly improves the precision of the surgery. This results in a potential reduction of surgical time, which has significant cost implications for all parties involved. ${ }^{7}$ The South African public health sector faces multiple challenges. It has long arthroplasty waiting lists as a result of surgical skill shortages and severe financial resource constraints. The value of having a tool like pre-operative templating available, which reduces surgical time and increases theatre efficiency while saving on long-term costs, is significant (Figure 3).

Our results were comparable to that of Gamble et al. ${ }^{6}$ who, with the use of digital pre-operative templating, showed an accurate

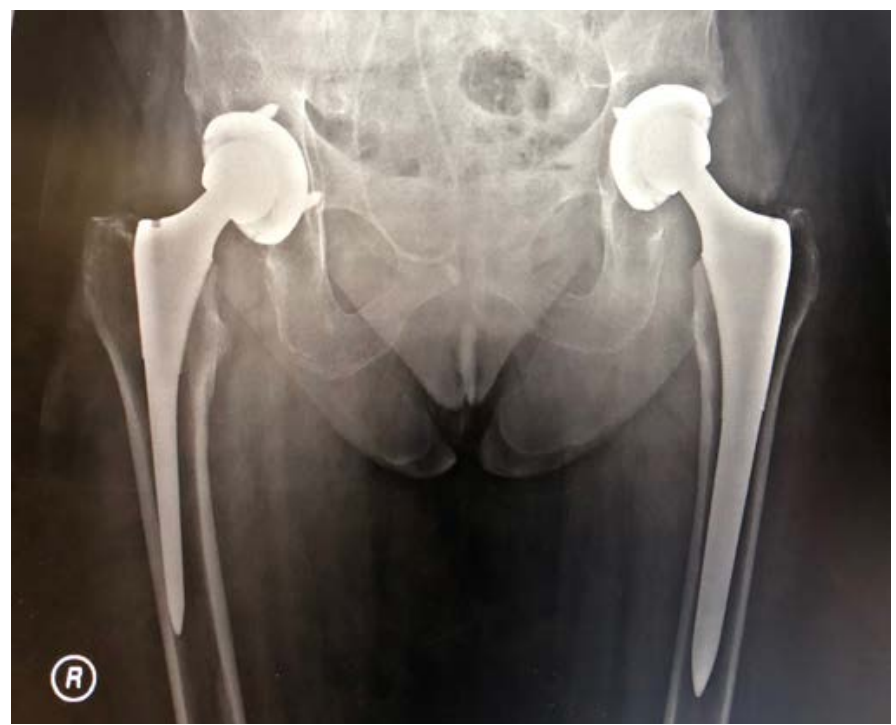

Figure 3. AP pelvis radiograph showing a recruited patient with the implant inserted on the right without the use of pre-operative templating, and the implant on the left with the use of pre-operative templating. The right hip implant is undersized, which could have significant long-term implications. 
prediction in $38 \%$ of cases in terms of acetabular cup sizing (29\% in our study), and $35 \%$ of cases in terms of femoral stem component sizing (30\% in our study). Accuracy improved dramatically to $80 \%$ (71\% in our study) for acetabular cup sizing and $85 \%$ (79\% in our study) for femoral stem sizing, when cases within one implant size above or below what was templated were included. Considering that their study was conducted in a high-income, high-volume arthroplasty unit, our study shows that pre-operative digital templating in a low-volume, resource-constrained unit can be just as accurate and valuable.

Reasons for templating implant outliers (implant size bigger or smaller than two sizes from the templated size) in our dataset may have been due to human error in the templating process during the initiating stage of implementing pre-operative digital templating. Surgeon experience in a low-volume unit that uses the digital templating software may also have an effect on the accuracy and reliability of templating. Even with the technique being carefully applied, patient body habitus, anatomical variability, and radiograph quality may become sources of error in pre-operative templating. ${ }^{15,16}$

Body mass index (BMI) was not measured as part of this study. In South Africa we have the second largest female population in Africa. ${ }^{17} \mathrm{~A}$ high BMI may contribute to a bigger margin of error in digital templating. This may have been the possible reason for the female predominance in our outlier group.

Our study has a few limitations. Firstly, this study was conducted in a low-volume arthroplasty unit with only one or two elective THA cases being performed per week. These cases were not necessarily done uncemented, thus the numbers were small over the review period. Reasons for outliers could be due to templating errors possibly as a result of implementation of digital templating, combined with inexperience of junior orthopaedic staff. Intraand inter-observer reliability and professional experience were not specifically analysed during the templating process, and leg length discrepancy correction was not quantified pre- and postoperatively.

\section{Conclusion}

The benefits of pre-operative templating have been shown in numerous studies conducted in high-volume arthroplasty units in high-income countries. The results of this study, conducted in a lowvolume, resource-constrained orthopaedic unit, are promising and comparable in accuracy to other published literature. This study confirms the multiple advantages of having digital pre-operative templating available for any orthopaedic unit that preforms THA and we strongly advocate the use of digital pre-operative templating, done in a stepwise technique, for any surgeon that performs THA currently.

\section{Ethics statement}

The authors declare that this submission is in accordance with the principles laid down by the Responsible Research Publication Position Statements as developed th the 2nd World Conference on Research Integrity in Singapore, 2010. Prior to commencement of the study ethical approval was obtained from the following ethical review board: Stellenbosch University Health Research Ethics Committee (HREC) Reference Number: \#6534. All procedures were in accordance with the ethical standards of the responsible committee on human experimentation (institutional and national) and with the Helsinki Declaration of 1975 , as revised in 2008. Informed written consent was not obtained from all patients for being included in the study.

\section{Declaration}

The authors declare authorship of this article and that they have followed sound scientific research practice. This research is original and does not transgress plagiarism policies.

\section{Author contributions}

KRW contributed to the conception and design of the work; the acquisition, analysis and interpretation of data for the work; drafting the work and final approval of the version to be submitted to the journal.

FWK contributed to the acquisition, analysis and interpretation of data for the work and assisted with drafting the work.

$\mathrm{CAB}$ contributed to the conception and design of the work.

TF contributed to the final approval of the version to be submitted to the journal.

JDJ contributed to drafting the work, revising it critically for important intellectual content, and final approval of the version to be submitted to the journal.

\section{ORCID}

Wiese KR (D) http://orcid.org/0000-0003-1764-8500

Kock FW (D) http://orcid.org/0000-0003-3943-1994

Blake CA (D) http://orcid.org/0000-0002-3592-4823

Franken T (D) http://orcid.org/0000-0003-0033-8303

Jordaan JD (iD http://orcid.org/0000-0002-6150-9463

\section{References}

1. Kahlenberg C, Nwachukwu BU, Schairer WW, Steinhaus ME, Cross MB. Patient satisfaction reporting after total hip arthroplasty: a systematic review. Orthopedics. 2017;40(3):e400-e404. doi:10.3928/01477447-20170120-04

2. Davies P, Graham S, Maqungo S, Harrison W. Total joint replacement in sub-saharan africa: a systematic review. Tropical Doctor. 2019;49(2):120-28. doi: 10.1177/0049475518822239.

3. Unnanuntana A, Wagner D, Goodman SB. The accuracy of preoperative templating in cementless total hip arthroplasty. $J$ Arthroplasty. 2009;24(2):180-86. doi:10.1016/j.arth.2007.10.032.

4. Tripuraneni KR, Archibeck MJ, Junick DW, Carothers JT, White RE. Common errors in the execution of preoperative templating for primary total hip arthroplasty. J Arthroplasty. 2010;25(8):1235-39. doi:10.1016/j.arth.2009.10.004.

5. Müller M. Lessons of 30 years of total hip arthroplasty. Clin Orthop Relat Res. 1992 Jan;274:12-21.

6. Gamble P, De Beer J, Petruccelli D, Winemaker M. The accuracy of digital templating in uncemented total hip arthroplasty. J Arthroplasty. 2010;25(4):529-32. doi:10.1016/j.arth.2009.04.011.

7. Blake C, Van der Merwe J, Raubenheimer J. A practical way to calibrate digital radiographs in hip arthroplasty. SA Orthop J. 2013;12(4):33-37.

8. Sinclair VF, Wilson J, Jain NPM, Knowles D. Assessment of accuracy of marker ball placement in pre-operative templating for total hip arthroplasty. J Arthroplasty. 2014;29(8):1658-60. doi:10.1016/j.arth.2014.03.013.

9. Katz J, et al. Association of hospital and surgeon volume of total hip replacement with functional status and satisfaction three years following surgery. Arthritis Rheum. 2003;48:560-68. doi:10.1002/ art.10754.

10. Scheerlinck T. Primary hip arthroplasty templating on standaard radiographs. A stepwise approach. Acta Orthop Belg. 2010;76:432-42.

11. Bono J. Digital templating in total hip arthroplasty. $J$ Bone Joint Surg. 2004;86:118-22. doi: 10.2106/00004623-200412002-00016.

12. Laucis N, Chowdhury M, Dasgupta A, Bhattacharyya T. Trend toward high-volume hospitals and the influence on complications in knee and hip arthroplasty. J Bone Joint Surg. 2016;98:707-12. doi:10.2106/JBJS.15.00399.

13. Anyaehie U, Eyichukwu G, Nwadinigwe C. Total knee replacement in a resource constrained environment: a preliminary report. Niger J Clin Pract. 2017;20:369-75. doi:10.4103/1119-3077.196117.

14. Knight J, Atwater R. Preoperative planning for total hip arthroplasty. Quantitating its utility and precision. J Arthroplasty. 1992; 7(Supp):403-409.

15. Hamilton WG. The rationale for a collared titanium stem in primary total hip arthroplasty. DePuy Synthes, 2017.

16. McLawhorn A, Nam D, McArthur B, Cross M, Su E. Preoperative templating and its intraoperative applications for hip resurfacing arthroplasty. Am J Orthop. 2012;41:E157-62.

17. Puoane T, Steyn K, Bradshaw D, Laubscher R, Fourie J, Lambert V, Mbananga N. Obesity in South Africa: The South African Demographic and Health Survey. Obesity Research. 2002;10:1038-48. doi:10.1038/oby.2002.141 